\title{
Test of Micro-pattern Gaseous Detector modules with a large prototype Time Projection Chamber
}

\author{
Deb Sankar Bhattacharya ${ }^{\text {粒 }}$ \\ CEA/Irfu Saclay, 91191 Gif sur Yvette cedex (FR) \\ E-mail: deb.sankar.bhattacharya@cern.ch
}

The International Large Detector (ILD) is one of the detector concepts at the ILC. The central tracking is planned to be based on a Time Projection Chamber (TPC). Research and development for a Micro-pattern Gaseous Detector (MPGD) TPC has been performed within the framework of LCTPC collaboration. Beam tests have been carried out using a large prototype, equipped with up to seven identical modules in a $1 \mathrm{~T}$ magnetic field at DESY. Results obtained with two technologies (GEM, Resistive Micromegas) are presented. Recently a new resistive material, diamond-like carbon, has been tested and compared with more usual carbon-loaded polyimide. Theses tests demonstrate that a MPGD TPC can be used in the tracking at ILC. Two-phase $\mathrm{CO}_{2}$ cooling has been applied to reduce temperature of the readout electronics.

The European Physical Society Conference on High Energy Physics

22-29 July 2015

Vienna, Austria

\footnotetext{
*On behalf of LCTPC collaboration.

†peaker.

${ }^{\ddagger}$ Now at SINP, Kolkata, India.
} 


\section{Introduction}

The International Linear Collider (ILC) [1] is a discovery and precision measurement machine where $e^{-}$and $e^{+}$collide at a centre-of-mass energy in the $240-1000 \mathrm{GeV}$ range. It provides opportunities to measure Higgs couplings, including its self-coupling. The main Higgs production mechanism is associated with $\mathrm{Z} \mathrm{H}$ production, called Higgs-Strahlung. An unbiased sample of Higgs decay can be selected by tagging the recoiling $\mathrm{Z}$ in its leptonic decays, allowing precision measurements of the mass and branching fractions.

The International Large Detector (ILD) [2] is one of the two detector concepts for the ILC. The central tracking at ILD is based on a Time Projection Chamber (TPC) [3]. The ILD-TPC, which is planned to run in a $3.5 \mathrm{~T}$ magnetic field, will be $4.6 \mathrm{~m}$ in length and $3.6 \mathrm{~m}$ in diameter. The physics measurements at ILC require excellent pattern recognition and single point resolution less than 100 micron over the full drift length of the TPC. Micro-pattern Gaseous Detectors (MPGDs) [4] are known for their good position resolution. Two MPGDs, Micromegas [5] and GEM [6], are being tested with a Large Prototype of a TPC (LP-TPC) [7]. The performances of the two detectors are discussed here.

\section{Large Prototype of a TPC}

A large prototype of a TPC for ILC is installed at DESY since 2008 [7]. The field cage has the inner length of $56.80 \mathrm{~cm}$ and inner diameter of $72 \mathrm{~cm}$. It is placed inside a solenoidal magnet which produces a field up to $1.2 \mathrm{~T}$ ( $1 \mathrm{~T}$ in most of the data presented here). The whole magnet sits on a movable stage, which allows translation of the TPC along its drift axis and in the vertical direction. It can also rotate in the horizontal plane, and the field cage can be rotated by hand around its axis in the magnet to explore different azimuthal angles of the beam. Up to seven MPGD modules can be mounted on the endplate. In cases where the endplate is partially equipped, dummy modules are inserted in the available sites to complete the equipotential end plane. Micromegas (Fig. 1a) and GEM (Fig. 1b) modules have the same 'keystone' shape and identical size of about $22 \mathrm{~cm}$ width and $17 \mathrm{~cm}$ height.

\subsection{Resistive Micromegas}

In resistive Micromegas [8] the anode plane is covered with a thin layer of resistive material on top of an insulator. This constitutes a resistive-capacitive continuous layer which spreads the charge of the ionization clusters, so that signal is caught and shaped over several pads. The position of the track is obtained by fitting the pad response function to the set of charge depositions on each pad row. Eventually, this technique also helps to reduce the number of pads, and thus of electronic channels.

It is also very efficient to reduce the spark rate and their intensity. This allowed to remove the channel protection, saving a large fraction of space in front-end readout cards. The read out of Micromegas module is segmented in 1726 pads of size $3 \mathrm{~mm} \times 7 \mathrm{~mm}$ and arranged in 24 rows. Micromegas with different resistive materials have been tested at the LP-TPC. Usually Carbon Loaded Kapton (CLK) modules were used at the LP-TPC. In 2015, two new Diamond Like Carbon 
(DLC) modules were prepared and applied at the beam test which allows to perform a comparative study.

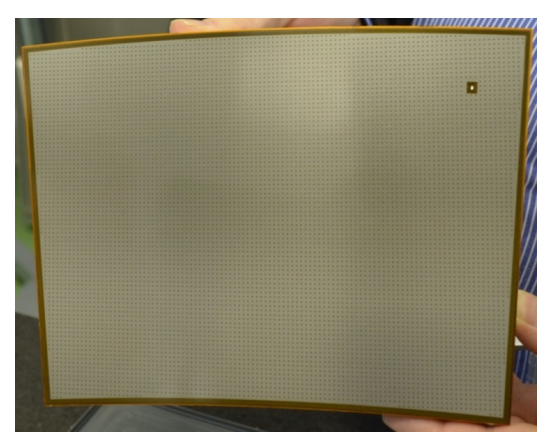

(a)

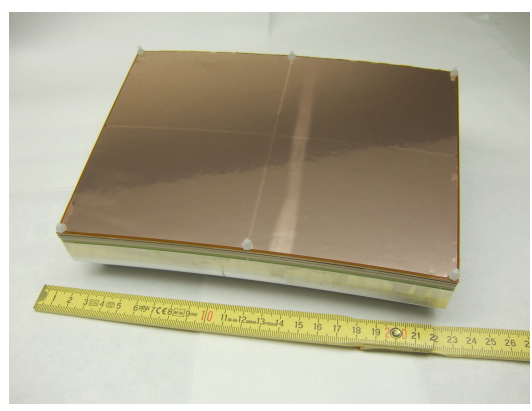

(b)

Figure 1: Two MPGD modules for the LP-TPC. (a) Resistive Micromegas module with 1726 channels (b) GridGEM module with 4839 channels

\subsection{Gas Electron Multiplier}

The GridGEM consists of a stack of three GEMs [9]. The distance of separation between the GEMs are: $\mathrm{d}_{\text {induction }}=3 \mathrm{~mm}, \mathrm{~d}_{\text {transfer }}=2 \mathrm{~mm}$. Using three GEMs helps to collect ions that can flow back to the sensitive volume of the TPC creating non uniformity in electric field. A stack of three GEMs also allows a stable high gain to be maintained. The anode is segmented in 4839 pads of size $1.26 \mathrm{~mm} \times 5.85 \mathrm{~mm}$ and arranged in 28 rows. After the amplifications in three steps, the clusters can fire up to 4.5 pads on average.

\subsubsection{Induced discharges in GEM}

The GEM foil is segmented in four parts to ensure high voltage stability. Two thin, light weight ceramic bars maintain the flatness. This ensures field uniformity. Measured flatness is below 100 micron undulations. Under special conditions discharges are observed in different sectors. The reason for these discharges are now studied. A single trip in one sector may trigger a second trip in another sector. Only a combination of multiple trips poses a threat to destroy the GEM. The number of multiple trips is counted by a $640 \times 480$ pixel webcam. It receives lights from the trips and integrates over $33 \mathrm{~ms}$. All the noisy pixels are set to 0 . A threshold of 150 is set for the signal. All active pixels are added up. In case of multiple trips, more pixels are fired and the magnitude of the signal intensity gets higher. Three separate intensity peaks are populated for single, double and triple trips respectively in figure $2 \mathrm{a}$. The counts for multiple trips are less than the single trip. Common ground of all different sectors is identified as a probable reason for this. Further study is going on.

\section{Results}

\subsection{Drift velocity and time zero determination}

Determination of drift velocity of the electrons in the TPC gas is a basic measurement for detailed analysis. At a given drift field, the beam position is varied at different drift lengths $(Z)$. 
The corresponding drift times are reconstructed from the primary analysis. When the different beam positions, which are basically the TPC-table readings, are plotted against the corresponding reconstructed time, the slope of the straight line fit gives the drift velocity. Two different lines for two different fields intersect when extrapolated towards the origin. The point of intersection corresponds to a lower limit on the electronic time scale. There does not exist any drift before this time. This limit, also referred as $T_{0}$ is used to calibrate the entire electronic time scale. For GEM, (figure 2b) drift velocities are measured to be $77.26 \pm 0.04 \mu \mathrm{m} / \mathrm{ns}$ and $55.12 \pm 0.03 \mu \mathrm{m} / \mathrm{ns}$ for the drift fields $240 \mathrm{~V} / \mathrm{cm}$ and $130 \mathrm{~V} / \mathrm{cm}$ respectively. Similar measurement is also performed with Micromegas.

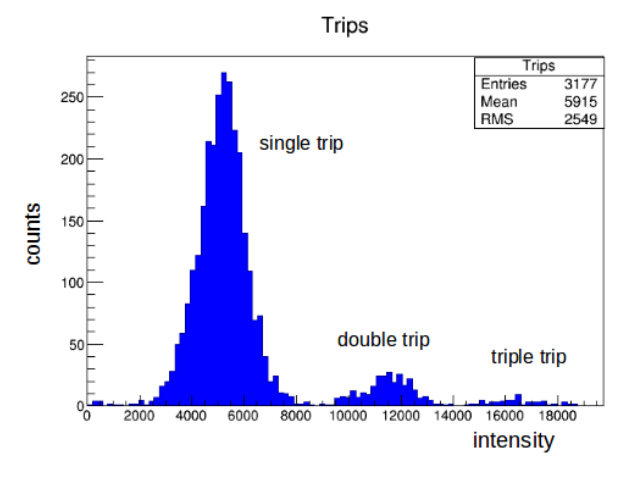

(a)

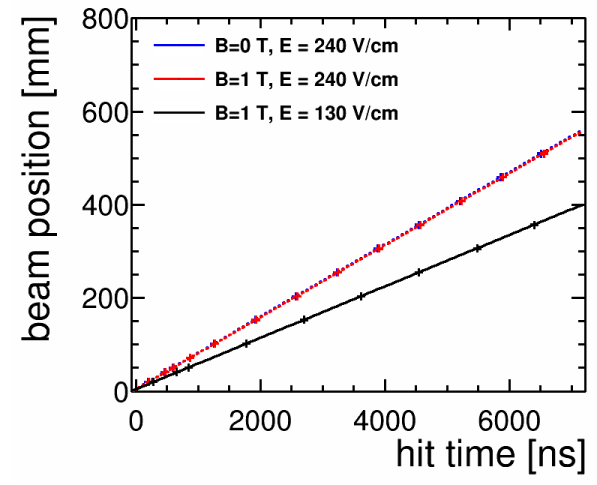

(b)

Figure 2: (a)The peaks from the left corresponds to single, double and triple trips respectively. A $640 \times 480$ pixel webcam receives light signal from the trips and integrates over $33 \mathrm{~ms}$. For multiple trips, more pixels are fired and signal contributes to higher intensity peaks in $\mathrm{X}$ axis. Y axis is count. (b) Drift velocity measurement with the GEMs. The beam position is plotted against the reconstructed hit times. The slope gives the drift velocity. The intersection point of two curves, which comes from two different fields, gives the $\mathrm{T}_{0}$.

\subsection{Alignment and Distortion corrections}

There are two main challenges when reconstructing the track: misalignment of the modules and inhomogeneous electric field near the edge of the detector. During the commissioning of the modules at the end-plate, there is a chance that they are slightly misaligned. Due to the absence of any external reference, it's not directly possible to find out the misalignment. The geometry of the detectors is input to the analysis framework (MarlinTPC[10]) through a geometry file [11]. Taking the central module as an arbitrary reference, very small rotational or translational $(60 \mu \mathrm{m})$ corrections are gradually applied to the geometry file through 'miliped' [12]. It takes few iterations to converge to the final corrected version of the geometry file. Figure 3a shows distribution of residual for individual rows, before and after the alignment correction.

The distortion effect comes due to the fact that the electric field is not uniform near the edges of the modules. In case of Micromegas, the peripheral frame is on ground potential. For GEM, the peripheral frame is kept in a relatively low potential. This causes the field lines to converge on the low potential regions which deviates the drifting electrons towards the edges. As a result, the reconstructed track appears to be distorted near the boundaries of the modules. As the field lines at low drift remains no longer parallel to the TPC axis, in presence of magnetic field, the electrons 


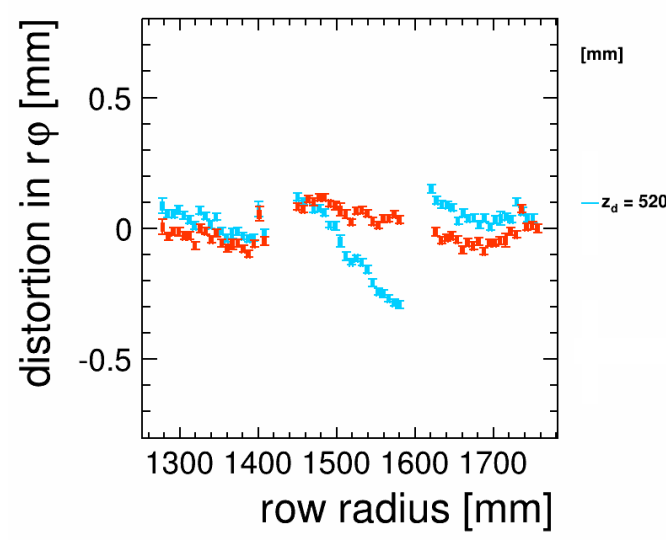

(a)

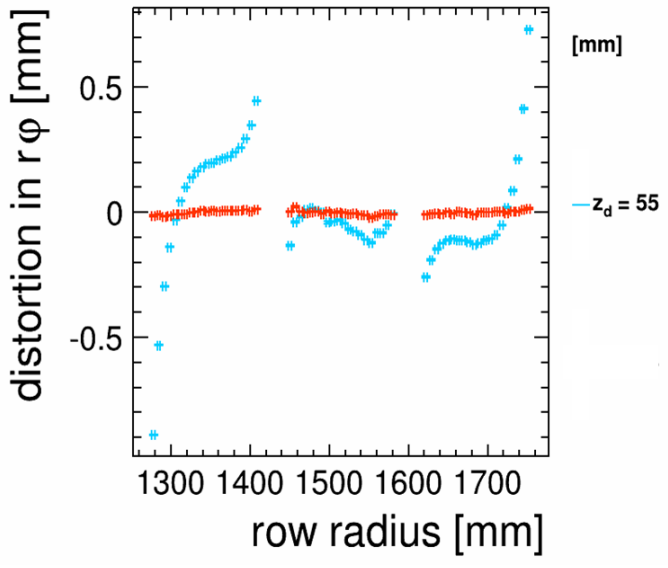

(b)

Figure 3: Alignment and field distortion correction in Micromegas. (a) Alignment correction is done at B $=0$ T. The blue residuals are before corrections where the red ones are done after the alignment correction is made. (b) Electric field distortion correction is done at $\mathrm{B}=1 \mathrm{~T}$. The $\mathrm{E} \times \mathrm{B}$ effect can be seen near the edges. The blue residuals are before corrections while the red ones are done after the distortion correction is accomplished.

experience a force due to the $\mathrm{E} \times \mathrm{B}$ effect and the track obtains a ' $\mathrm{S}$ ' shape near the edge. The average residual of individual rows is calculated and corrected during analysis in MarlinTPC [10]. Figure $3 \mathrm{~b}$ shows the distribution of average residual before and after the distortion correction in presence of magnetic field.

\section{3 r-phi resolution and $\mathrm{Z}$ resolution}

The space resolution is defined by: $\sigma=\sqrt{\Delta_{i n} \cdot \Delta_{e x}}$, where the residual of a hit is defined as, $\Delta=X_{\text {hit }}-X_{\text {track }}$. The $\Delta_{\text {in }}$ of a hit refers to the residual when the hit-position is included in the track fitting and the $\Delta_{e x}$ refers to the residual when the hit is excluded from track fitting. The position resolution also depends on: drift distance $(\mathrm{Z})$, effective number of electrons $\left(N_{e f f}\right)$, coefficient of transverse diffusion $\left(C_{d}\right)$. The relation [13] is given by:

$$
\sigma=\sqrt{\sigma_{0}^{2}+\frac{C_{d}^{2} \cdot Z}{N_{e f f}}}
$$

where $\sigma_{0}$ is the resolution for $Z=0$ and it depends on the technology of the detector. The dependence of space resolution on drift distance for both Micromegas and GEM is shown in Figure 4a and $4 \mathrm{~b}$ respectively. The physics requirements set the goal of $100 \mu \mathrm{m}$ of $\mathrm{r}-\phi$ resolution in $2.3 \mathrm{~m}$ drift for a 3.5 T magnetic field. At the LP-TPC, in a 1 Tesla magnetic field, the resolution within $60 \mathrm{~cm}$ drift distance corresponds to the ILD requirements using an extrapolation based on the calculated diffusion coefficient. The $\mathrm{Z}$ resolution at different drift distances is given in figure $5 \mathrm{a}$ and $5 \mathrm{~b}$ for Micromegas and GEM respectively. This also corresponds to (when extrapolated to $3.5 \mathrm{~T}$ ) the ILD requirement of $0.4 \mathrm{~mm}$ resolution at zero drift and $1.4 \mathrm{~mm}$ at full drift.

\subsection{Comparison of two different resistive Micromegas}

As already mentioned, the charge dispersion technique improves the position resolution of a Micromegas. The dispersion depends on the resistive coating. Previously, CLK was used as the 


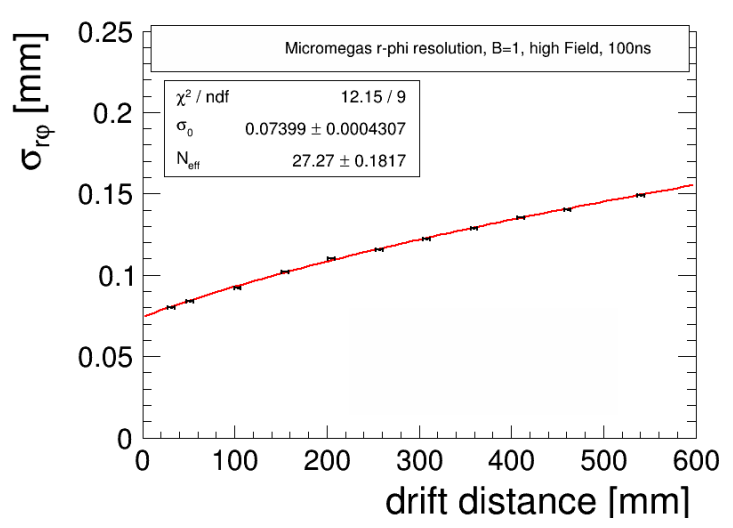

(a)

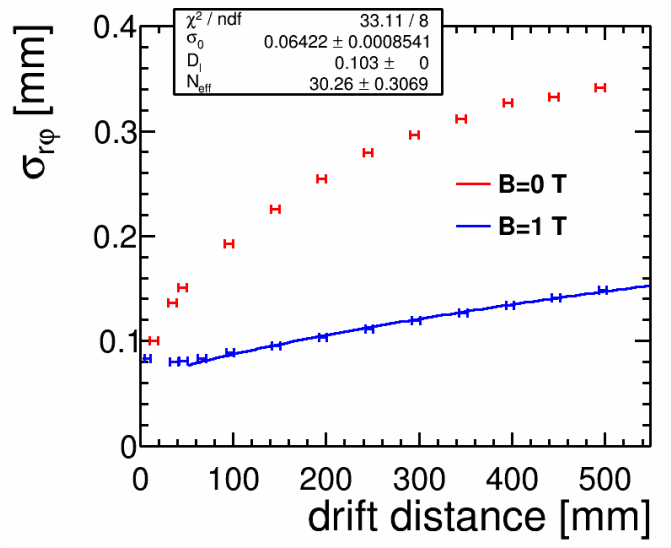

(b)

Figure 4: Dependence of space resolution on drift distance. (a) Micromegas at $\mathrm{B}=1 \mathrm{~T}$. (b) GEM at $\mathrm{B}=0 \mathrm{~T}$ and $\mathrm{B}=1 \mathrm{~T}$.

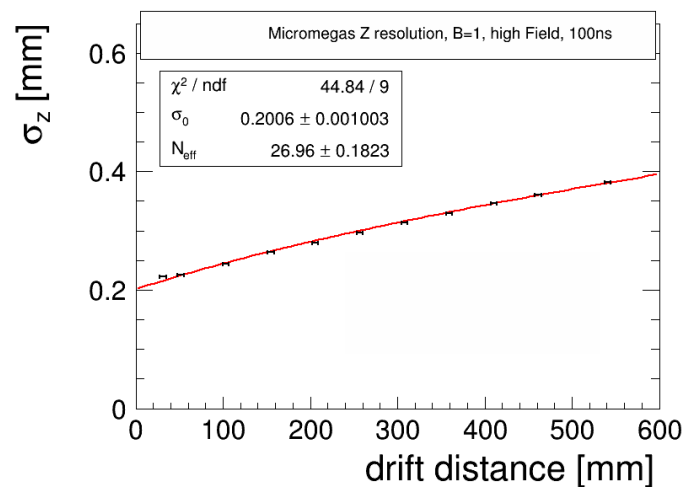

(a)

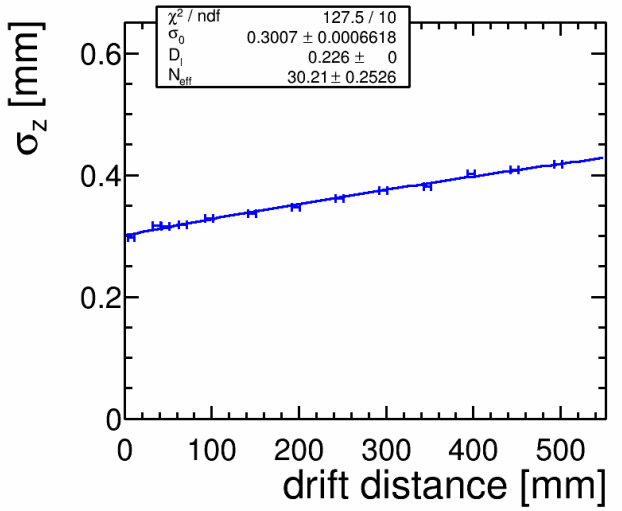

(b)

Figure 5: Variation of spatial resolution along drift distance with drift distance. (a) Micromegas at B $=1 \mathrm{~T}$. (b) GEM at $\mathrm{B}=1 \mathrm{~T}$.

resistive layer. Due to the unavailability of the CLKs (from USA), the DLC is imported from Japan. The DLC modules are prepared and tested at CERN and then used in the 2015 beam test. A quantitative comparison is made between the CLK and DLC modules in figure 6. The charge dispersion is seen to be slightly large in DLC. Figure 6a shows two histograms; the red one is for DLC where the blue is for CLK. Each of the histograms corresponds to the average number of pads fired when a charge cluster hits the anode. For DLC, the number is 4.33 where for CLK the number is 3.13 .

In figure $6 \mathrm{~b}$, the row-wise resolution for all the three MM modules are shown. The different sets (colors) are for different drift distances. The three bands represent three modules. The first one (module 0) is a DLC module while the rest are the CLKs. It may be noticed that the row wise resolution of the DLC module is very much comparable to the CLK modules. This implies efficient performance of the DLCs during the unavailability of the CLKs. Other advantages of the DLC modules are that they are robust and produced in Japan (where the ILC is very likely to be built). 


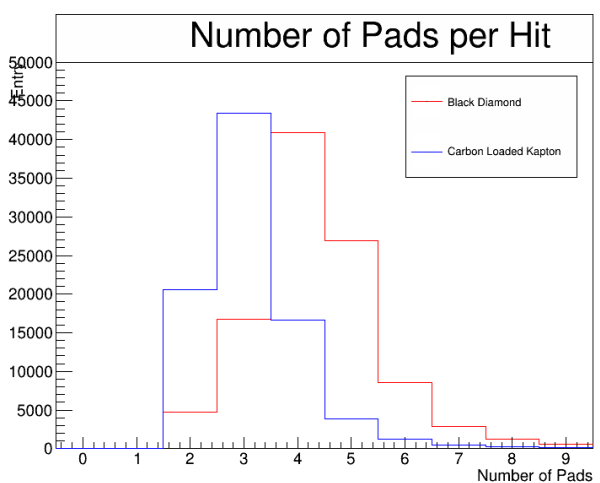

(a)

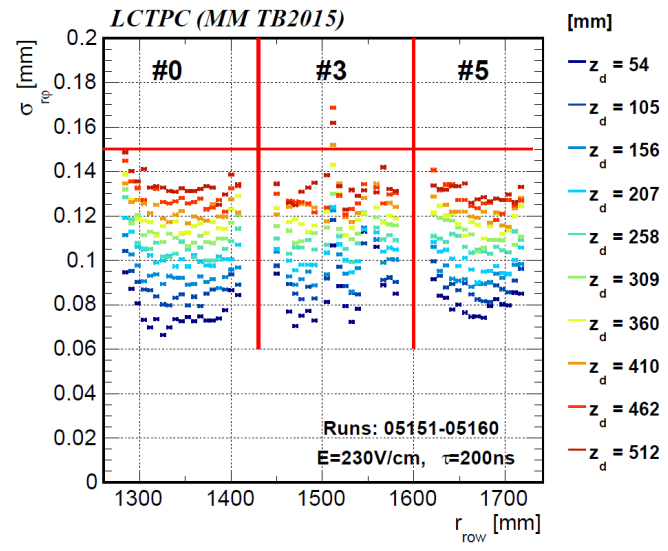

(b)

Figure 6: Comparison of CLK and DLC (Black Diamond) Micromegas modules. Module-0 is a DLC, where module-3 and module5 are CLKs. (a)'Pads per Hit' for the DLC and CLK are 4.33 and 3.13 respectively. (b) Row by row r-phi resolution comparison of three Micromegas modules.

\subsection{Two-phase $\mathrm{CO}_{2}$ cooling}

The readout electronics of a Micromegas (MM) module consumes about $26 \mathrm{~W}$ of electric power. This causes the temperature of the electronic board to increase up to $70^{\circ} \mathrm{C}$. Increase in temperature results in damage of the electronics. The development of a temperature gradient in the Time Projection Chamber (TPC) may affect a precise measurement. A two-phase $\mathrm{CO}_{2}$ cooling has been applied to remove heat from the MM modules during two test beam experiments at DESY, Hamburg. The application of Two-phase $\mathrm{CO}_{2}$ keeps the temperature below $30^{\circ} \mathrm{C}$ and stabilized within $0.2^{\circ} \mathrm{C}$. The reason why $\mathrm{CO}_{2}$ cooling is preferred is that the cooling happens during the phase change when temperature of the system remains constant. This ensures uniform and stable cooling. Two phase $\mathrm{CO}_{2}$ cooling was applied for all seven Micromegas modules during the 2015 beam test. Figure 7 shows the temperature of individual FECs and the FEM of a single module during the experiment. Comprehensive study of the whole process is done by simulation. Details of experiments and simulations on two-phase $\mathrm{CO}_{2}$ cooling for Micromegas can be found here [14].

\section{Conclusion}

A large prototype of a TPC has been installed in 2008 at DESY, Hamburg, and two different technologies, Micromegas and GEM, have been tested successfully. A new kind resistive material, Diamond Like Carbon, has been tested for resistive Micromegas technology in March 2015. It shows comparable performances with the Carbon Loaded Polyimide and its purchase is easier. A detailed study on GEM flatness and multiple discharge problem has been carried out. Problems occurring due to misalignment of the modules and localized electric filed distortion near the edges of the detectors have been studied in detail and corrected during analysis. The resolution has been measured to be below $80 \mu \mathrm{m}$ at zero drift distance for both technologies and evolves with drift distance as expected from diffusion. This, extrapolated to a $2.3 \mathrm{~m}$ drift length and in a $3.5 \mathrm{~T}$ 


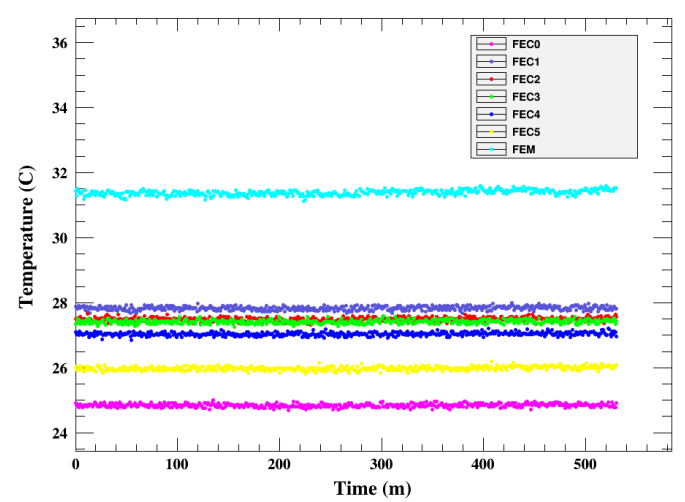

Figure 7: Temperature profile of all the six Front End Cards and the Front End Mezzanine of a Micromegas module when two-phase $\mathrm{CO}_{2}$ cooling was applied during the 2015 beam test.

magnetic field, meets the requirements of roughly $100 \mu \mathrm{m}$ resolution. The $\mathrm{Z}$ resolution in $1 \mathrm{~T}$ magnetic field also corresponds to the ILD requirement. A two-phase $\mathrm{CO}_{2}$ cooling has been used uninterruptedly for more than $80 \mathrm{hrs}$ for Micromegas. Temperature of individual Front End Cards (FECs) is kept below $30^{\circ} \mathrm{C}$ and stable within $0.2^{\circ} \mathrm{C}$ during the beam test. The tests have shown that both technologies can meet the ILD requirements. The Micromegas test is a 'proof of existence' of a realistic integration solution, as far as electronics and cooling are concerned.

\section{Acknowledgments}

The LCTPC collaboration thanks the DESY management for its hospitality and KEK, Japan for financing the $\mathrm{CO}_{2}$ cooling system, TRACI. This work is partially financed by CEFIPRA/IFCPAR (Project 4304-01), EUDET and AIDA.

\section{References}

[1] T.Behnke, (ed.) et al., International Linear Collider Technical Design Report: Executive Summary 1 (2013).

[2] T.Behnke, (ed.) et al., International Linear Collider Technical Design Report: Detectors 4 (2013).

[3] H.J.Hilke, Time projection chamber, 2010 Rep. Prog. Phys. 73116201

[4] A. Oed, Micro pattern structures for gas detectors, Nucl. Instr. and Meth. A 471 (2001) 109-114.

[5] Y. Giomataris et al, MICROMEGAS: a high-granularity position-sensitive gaseous detector for high particle-flux environments, Nucl. Instrum. Methods A. 376 (1996) 29.

[6] F. Sauli, GEM: a new concept for electron amplification in gas detectors, Nucl. Instrum. Methods A. 386 (1997) 531.

[7] Thesis of Peter Schade : 'Development and Construction of a Large TPC Prototype for the ILC and Study of $\tau$ Polarisation in $\tau^{s}$ Decays with the ILD Detector', UniversitätHamburg, 2009.

http: //www-library.desy.de/cgi-bin/showprep.pl?desy-thesis-09-040 
[8] M.S.Dixit et al, Simulating the charge dispersion phenomena in Micro Pattern Gas Detectors with a resistive anode, Nucl. Instrum. Methods A. 566 (2006) 281-285.

[9] Thesis of Klaus Zenker:'Studies of field distortions in a Time Projection Chamber for the International Linear Collider', UniversitätHamburg, 2014.

http: / / www-library.desy.de/cgi-bin/showprep.pl?desy-thesis-14-044

[10] MarlinTPC: http://ilcsoft.desy.de/portal/software_packages/marlintpc/

[11] Thesis of Wenxin Wang: 'A Large Area Micromegas TPC for Tracking at the ILC', University Paris - Sud, 2013.

[12] Miliped: http://www.desy.de/ blobel/mptalks.html

[13] D.C. Arogancia et al, Study in a beam test of the resolution of a Micromegas TPC with standard readout pads, Nucl. Instrum. Methods A. 602 (2009) 403-414.

[14] D.S. Bhattacharya et al, Measurement and simulation of two-phase CO2 cooling in Micromegas modules for a Large Prototype of Time Projection Chamber, JINST. 10 (2015) P08001. 